\title{
GOLIA: an INTEGRAL archive at INAF-IASF Milano
}

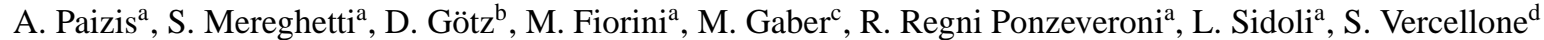 \\ ${ }^{a}$ INAF-IASF, Sezione di Milano, Via Bassini 15, 20133 Milano, Italy \\ ${ }^{b}$ AIM/Service d'Astrophysique - CEA Saclay DSM/Irfu, Gif sur Yvette, France \\ ${ }^{c}$ Former ISDC, Chemin d'Ecogia 16, 1290 Versoix, Switzerland \\ ${ }^{d}$ INAF-IASF, Sezione di Palermo, Via Ugo La Malfa 153, 90146 Palermo, Italy
}

\begin{abstract}
We present the archive of the INTEGRAL data developed and maintained at INAF-IASF Milano. The archive comprises all the public data currently available (revolutions 0026-1079, i.e., December 2002 - August 2011). INTEGRAL data are downloaded from the ISDC Data Centre for Astrophysics, Geneva, on a regular basis as they become public and a customized analysis using the OSA 9.0 software package is routinely performed on the IBIS/ISGRI data. The scientific products include individual pointing images and the associated detected source lists in the 17-30,30-50, $17-50$ and $50-100 \mathrm{keV}$ energy bands, as well as light-curves binned over $100 \mathrm{~s}$ in the $17-30 \mathrm{keV}$ band for sources of interest. Dedicated scripts to handle such vast datasets and results have been developed. We make the analysis tools to build such an archive publicly available. The whole database (raw data and products) enables an easy access to the hard X-ray long-term behavior of a large sample of sources.
\end{abstract}

Keywords: Astronomical databases: miscellaneous, Gamma rays: general, Methods: data analysis, Surveys, X-rays: general

\section{Introduction}

The INTErnational Gamma-Ray Astrophysics Laboratory, INTEGRAL (Winkler et al., 2003), is a medium sized ESA mission successfully launched on October 17th, 2002. Its payload consists of two main gammaray instruments, the spectrometer SPI (Vedrenne et al., 2003) and the imager IBIS (Ubertini et al., 2003), covering the $15 \mathrm{keV}-10 \mathrm{MeV}$ band. IBIS is a high angular resolution gamma-ray imager optimized for accurate point source imaging and for the continuum and broad line spectroscopy. It consists of two layers, the lower energy one (IBIS/ISGRI, $15 \mathrm{keV}-1 \mathrm{MeV}$, key parameters in Table 1, Lebrun et al., 2003) and the higher energy one (IBIS/PICsIT, 0.175-10 MeV, Labanti et al., 2003). Co-aligned with SPI and IBIS are two X-ray monitors JEM-X (4-35 keV, Lund et al., 2003) and an optical monitor OMC (500-600 nm, Mas-Hesse et al., 2003). INTEGRAL nicely links the energy band of pointed soft X-ray missions (such as Chandra, XMMNewton) to high energy gamma ray space missions such as AGILE, Fermi and ground based $\mathrm{TeV}$ observatories.

Email address: ada@iasf-milano.inaf .it (A. Paizis)

Preprint submitted to Astronomy and Computing
Operating since 2002 and thanks to the synergy of its complementary instruments, INTEGRAL provides an impressive quantity of high quality data for the study of key science areas, such as nucleosynthesis, diagnostics of supernovae and supernovae remnants, compact sources (black holes, neutron stars, white dwarfs), electron-positron annihilation emission, super-massive black holes, the cosmic X-ray background, gamma ray bursts, etc (see Winkler et al., 2011, and references therein for a recent review). At the time of writing a total of about 8.5 years of data have become public and are available to the scientific community for exploitation.

INTEGRAL instruments have very wide fields of view. Even considering the fully coded field of view, where the signal to noise ratio is maximal, that is smaller than the total telescope field of view, we obtain fields of about $9^{\circ} \times 9^{\circ}$ for IBIS, $16^{\circ}$ (corner to corner) for SPI, $5^{\circ}(\varnothing)$ for JEM-X and $5^{\circ} \times 5^{\circ}$ for OMC. Hence a complete study of a given source (or class of sources) requires huge datasets to be downloaded each time.

In order to increment and ease our exploitation of INTEGRAL data, we undertook the task of preparing and maintaining an INTEGRAL archive, GOLIA (Gi- 
Table 1: Key parameters for IBIS/ISGRI (Winkler et al., 2003, 2011)

\begin{tabular}{cc}
\hline \hline Parameter & IBIS/ISGRI \\
\hline \hline Energy range & $15 \mathrm{keV}-1 \mathrm{MeV}$ \\
Detectors & $16384 \mathrm{CdTe}$ \\
Spectral resolution (FWHM) & $8 \mathrm{keV} \mathrm{@} 100 \mathrm{keV}$ \\
Field of view (fully coded) & $9^{\circ} \times 9^{\circ}$ \\
Angular resolution (FWHM) & $12^{\prime}$ \\
Source location (radius) & $1^{\prime}$ for $\mathrm{S} / \mathrm{N}=30$ \\
& $3^{\prime}$ for S/N=10 \\
Continuum sensit. @ $100 \mathrm{keV}$ & $2.9 \times 10^{-6} \mathrm{ph} \mathrm{cm}^{-2} \mathrm{~s}^{-1}$ \\
& $\left(3 \sigma\right.$ detection in $10^{5} \mathrm{~s}$ \\
Absolute timing accuracy $(3 \sigma)$ & $\Delta \mathrm{E}=\mathrm{E} / 2)$ \\
\hline \hline
\end{tabular}

ant On-Line INTEGRAL Archive). GOLIA's aims are twofold: provide a local database of the available public data to facilitate personalized analysis of already downloaded data, and offer easy-to-browse IBIS/ISGRI data products for a quick and efficient view of the hard $\mathrm{X}$ ray sky. All of which is locally and interactively available at INAF-IASF Milano. The scripts we have used to build GOLIA are publicly available, as described in Section 4.1.

\section{A walk through GOLIA}

The basic structure of GOLIA is shown in Fig. 1 and is described below.

\subsection{Building the archive (archive Owner)}

A detailed description of the INTEGRAL Off-line Scientific Analysis (OSA) software package, used to analyze INTEGRAL data, is beyond the scope of the paper and we refer to the Documentation web page $^{1}$ of the ISDC Data Centre for Astrophysics (former INTEGRAL Science Data Centre, Courvoisier et al., 2003). We recall here one key point: INTEGRAL data are organized into the so-called "Science Windows" (ScWs, that for the purposes of this work can be considered as corresponding to a pointing at a fixed sky position, $\sim 2 \mathrm{ks}$ ). During the scientific analysis, all the ScWs belonging to the same observation are grouped together to form an "Observation Group".

\footnotetext{
${ }^{1}$ http://www.isdc.unige.ch/integral/analysis\#Documentation
}

The OSA package performs tasks in a modular way, i.e. it first applies photon energy correction (COR level) to all the ScWs belonging to the Observation Group, then it performs the second step, the Good Time Interval computation (GTI) for all the ScWs, and so on. When the analysis of a single observation is to be done (e.g. a few days / weeks of data) this approach is efficient since it simplifies tasks such as mosaicking or light-curve collection. But since a global analysis of all the public data means handling several tens of thousands of pointings (currently 83733) we chose a single ScW based approach: each Observation Group consists of a single $\mathrm{ScW}$ and the analysis loops over all the available ScWs. Basically, the analysis is run at the level of the smallest available "data entity" (the $\mathrm{ScW}$ ), with no a-priori grouping chosen by the archive Owner. A-posteriori grouping of $\mathrm{ScWs}$, for mosaicking and light-curve collection, will then be possible as chosen by each archive User. Furthermore, if the analysis of one single $\mathrm{ScW}$ happens to fail (for any given reason, missing data, no GTI available, etc), the analysis will continue and be completed for the remaining ScWs, independently. The implementation of such a script can be done in many ways, in our case Shell scripts with a simple "foreach" loop have been chosen.

Our IBIS/ISGRI processing and post-processing steps (all performed by the archive Owner) are described below. All the scripts described in this section are publicly available, see Section 4.1.

1. Download: all the INTEGRAL public data are downloaded via the ISDC dedicated web page ${ }^{2}$.

2. Process: the standard OSA 9 software package ${ }^{3}$ is run on all public data of the IBIS/ISGRI instrument, looping over ScWs via the Shell scripts launch_IMA.sh and launch_LCR.sh. We decided to run the image analysis (IMA) on the whole archive first, to have a quantitative view of the results, and then run the light-curve extraction (LCR) part on it. The scripts can however be merged to perform IMA and LCR for each ScW. The following steps are performed:

2-1 Image reconstruction (IMA): for each pointing a standard analysis is performed in four energy bands: $17-30,30-50,17-50$ and $50-100 \mathrm{keV}$. The

\footnotetext{
${ }^{2}$ http://www.isdc.unige.ch/integral/archive\#DataRelease

${ }^{3}$ The latest version of the software package can be found at http://www.isdc.unige.ch/integral/analysis\#Software
} 


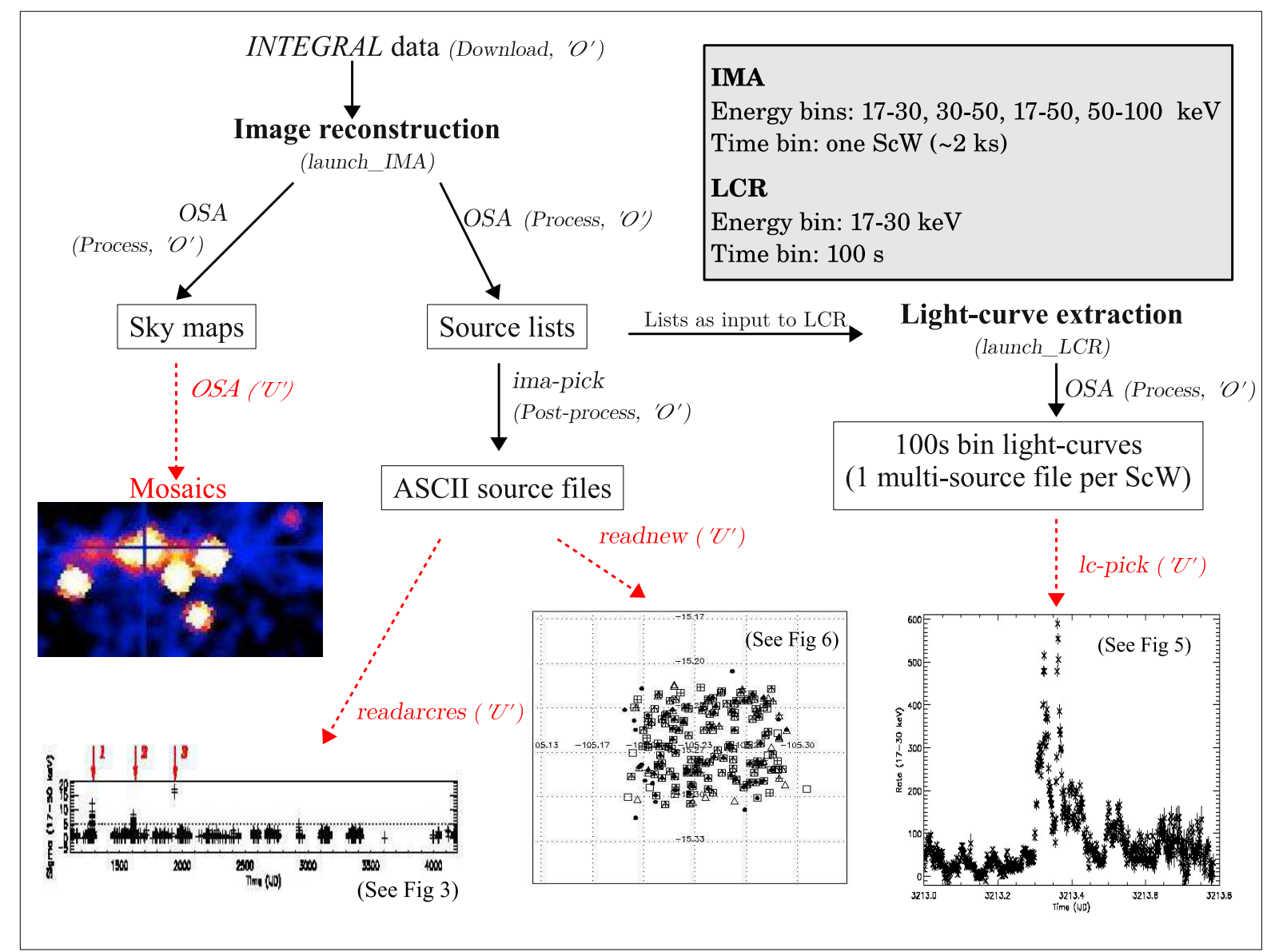

Figure 1: Basic structure of GOLIA. The black solid arrows show the steps made by the archive Owner (indicated as ' $O$ '), while the ones shown with dashed red arrows are performed by the archive User (indicated as ' $U$ '). The Download, Process and Post-process steps are shown. All the scripts of these three phases are publicly available (see Section 4.1). The tools launch_IMA, launch_LCR, ima_pick, readarcres and readnew have been developed specifically for our archive. See Section 2. 
results of this analysis are the starting point for pointing-based, $\sim 2 \mathrm{ks}$, light-curves (for all sources of the input catalogue) and maps/mosaics for a deeper analysis.

2-2 Catalogue creation: for each $\mathrm{ScW}$, a catalogue is created merging the bright sources discovered from the previous IMA step (with detection significance higher than 3) and sources of interest, regardless their detection level, be they new or already known. This allows to detect short bursts from interesting sources that would be otherwise missed in a single pointing ( $\sim 2 \mathrm{ks})$. Successful tests have been made on known GRBs.

2-3 Light-curve extraction (LCR): $17-30 \mathrm{keV}$ standard $100 \mathrm{~s}$ bin light-curve extraction is performed for all the sources of each $\mathrm{ScW}$ catalogue, i.e. thanks to step (2-2), also including sources of interest not necessarily detected in the $\sim 2 \mathrm{ks}$ image (step 2-1).

2-4 Clean and close down: relevant files are zipped and intermediate levels canceled.

3. Post-process: for each pointing, the IMA analysis step produces images in several bands and lists (FITS files) with position, flux and significance of the sources. Since map and source list files are derived for each $\mathrm{ScW}$, all the information about a given source is spread over many ScWs. The OSA 9 tool src_collect permits to collect IMA source results into a single table. However, it enables to retrieve results for one source at a time only and the results are saved in a FITS table. We have developed a PERL script (ima_pick.pl) that extracts in one go single ASCII files for all the sources present in the archive, one file per source. The tool can be run in a cumulative way (the ASCII files can be updated with the new incoming results). Each ASCII file contains the results of the imaging step and is the starting point for quick visualization by the Users.

We note that the ima_pick script collects results based on the source name, creating a SourceName.dat file. Every time the analysis software (OSA) detects a new source, the name $N E W_{-} 1$ is given (with incremental number for more new sources), hence in the whole catalogue we obtain several $N E W_{-}$, $N E W \_2$, etc., sources that are not the same, but have different positions. All these sources will be collected in the same
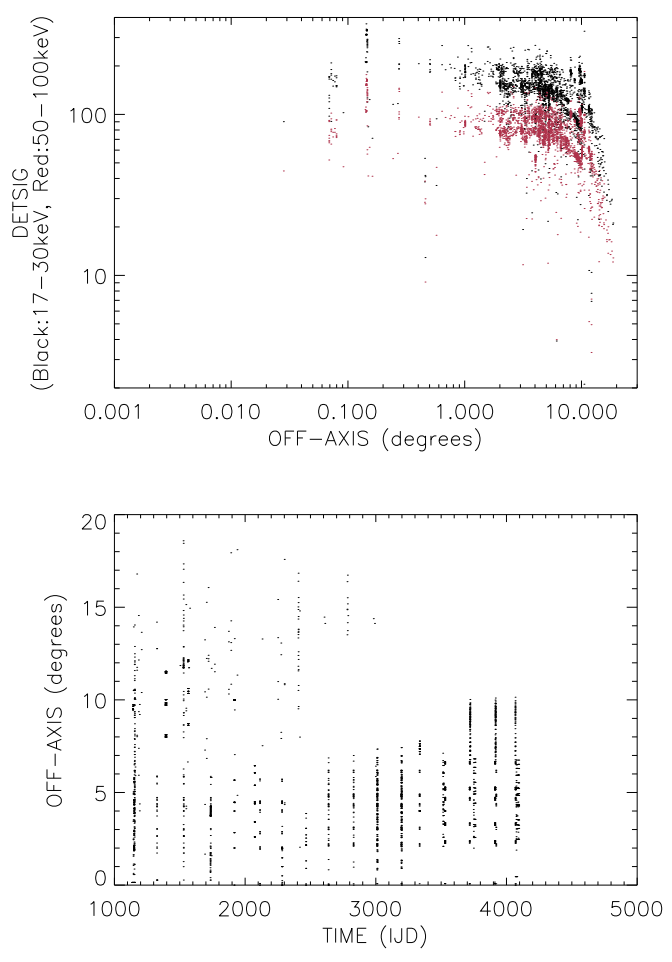

Figure 2: Crab detection significance and off-axis angle. Each point is a single $\mathrm{ScW}$, for a total of $2713 \mathrm{ScWs}$ with the Crab in the IBIS field of view (off axis angle $<20^{\circ}$, exposure time of about $4 \mathrm{Ms}$ ).

ASCII file by ima_pick and need to be disentangled based on their coordinates.

The introduction of the Post-process step, via the ima_pick tool (not part of the OSA package), leads to a huge simplification in the visualization of the image products. The produced ASCII files are a very easy-to-handle starting point and indeed whole-archive (8.5 year) light-curves can be extracted in matter of seconds for whichever source originally in the catalogue.

\subsection{Using the archive (archive User)}

Given the main steps performed by the archive Owner (Download, Process, Post-process), each User can access GOLIA in many ways. The mere Download, done by the Owner, allows the User to access the entire INTEGRAL public data for a personalized analysis, avoiding multiple time and space consuming downloads.

Once the Owner has completed the Process step, the User can already attain analyzed $\mathrm{ScW}$ images to build customized mosaics and source searches via a deeper 
analysis e.g. aimed to dim population studies. For brighter sources (or sources of interest) overall lightcurves binned at $100 \mathrm{~s}$ can be collected with the ISDC tool Ic_pick (see Fig. 5 and Section 3.3). The stacking of the Owner ScW-based products of the "Process" step (be they mosaics or $100 \mathrm{~s}$ bin light-curve collection) can be time consuming for the User if a very large number of ScWs is involved, as in standard OSA usage.

Finally, once all the key IMA parameters / results are saved in the ASCII file via the Post-process step, it is immediate to plot them in different ways to have a quantitative view of the database. As an example, in Fig. 2 we show the detection significance (DETSIG) of the Crab in the whole archive in two energy bands, as a function of the off-axis angle, and the evolution of the latter with time, expressed in INTEGRAL Julian Date, $\mathrm{IJD}^{4}$.

ASCII files are easily readable / plottable by several software tools. In our case, we have developed two IDL scripts (readarcres, readnew) to read and plot such files. The former tool allows us to handle / plot single source ASCII files, providing long-term light-curves in terms of count-rate or detection significance (as in Fig. 3 for the detection significance case). The latter (readnew) allows us to investigate the new sources enabling the search of NEW sources by coordinates. This allows us to select the occurrences of each NEW source in the whole archive (see Fig. 6 and Section 3.4).

\section{GOLIA: a starting point for new investigations}

In this section we give examples of published and work-in-progress scientific results based on GOLIA.

\subsection{Supergiant Fast X-ray Transients (SFXTs)}

Fig. 3 is an example of a whole-archive light-curve obtained with our interactive IDL readarcres script. The plot shows all the IBIS/ISGRI publicly available data of the transient IGR J11215-5952.

With such a long-term view, outbursts, trends, periodicities can be easily spotted. Indeed, the automatic pipeline running on public INTEGRAL observations present in our archive allowed us to discover that the Supergiant Fast X-ray Transient IGR J11215-5952 displays periodic outbursts (Sidoli et al., 2006). The source was discovered in 2005 (arrow 3 in Fig. 3; Lubiński et al., 2005), but thanks to our systematic re-analysis of INTEGRAL archival observations, we could discover two previously unnoticed outbursts (1 and 2 in Fig. 3 ),

\footnotetext{
${ }^{4} \mathrm{IJD}=\mathrm{MJD}-51544$
}


Figure 3: Whole-archive $\sim 2 \mathrm{ks}$ light-curve obtained for IGR J11215-5952. The plot shows all the INTEGRAL/IBIS public available data: 2442 pointings, $4.8 \mathrm{Ms}$, from revolution 0030 (Jan 2003, IJD 1107) to 1060 (Jun 2011, IJD 4190). Sigma $\geqq 5$ (horizontal line) is a detection.
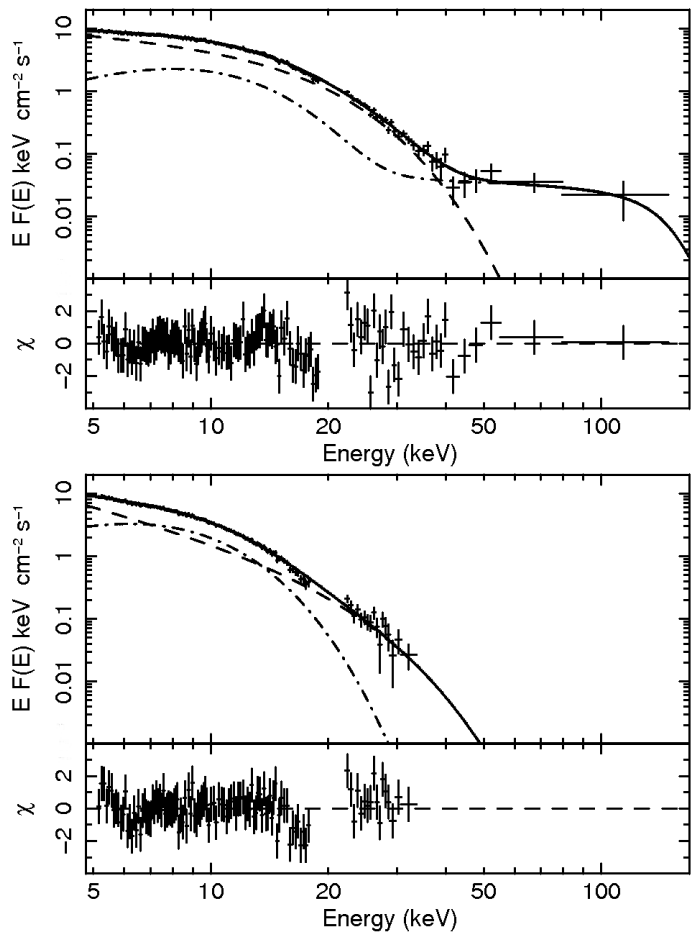

Figure 4: Unabsorbed $\mathrm{EF}(\mathrm{E})$ spectra with superposed best-fit wabs $\cdot($ compTB $[1]+$ compTB[2]) model (upper panels) and residuals in units of $\sigma$ between data and model (lower panels) for GX 5-1. Different line styles represent single components of the best-fit model. Dashed-line: pure thermal Comptonization for compTB[1]. Dash-dot line: bulk+thermal Comptonization (upper spectrum) or simple BB (lower one) for compTB[2]. Solid line: total spectrum (from Mainardi et al., 2010). 
spaced by intervals of 329 days, that occurred in July 2003 and May 2004, suggesting that this periodicity is the orbital period of the system. At that time we could not exclude that the true periodicity is half of this period (as indeed was later discovered, see Sidoli et al., 2007), because of the lack of INTEGRAL coverage of the source field of the first SFXT showing periodic outbursts.

This discovery triggered by our archive has led to further investigations within our team, including the development of a stellar wind model for OB supergiants to explain the transient and variable nature of SFXTs, and its application to all public INTEGRAL observations from 2003 to 2009 for a sample of 14 SFXTs (Ducci et al., 2009, 2010).

\subsection{Low Mass $X$-ray Binaries (LMXB)}

Visual inspection of light-curves such as Fig. 3 for persistently bright neutron star (NS) LMXBs (e.g. the Z source GX 5-1) has been the starting point for the systematic study of the evolution of the transient hard-tails in the spectra of these sources. Indeed hard tails dominate the X-ray emission above $30 \mathrm{keV}$, in an otherwise soft spectrum, and they result in a source detection in the 30-50 keV panel of Fig. 3 (e.g. Paizis et al., 2006a; Farinelli et al., 2008; Mainardi et al., 2010). An example for the transient tail can be seen in Fig. 4 for GX 5-1 (JEM-X and IBIS/ISGRI data). The origin of such tails dominating the spectra above $\sim 30 \mathrm{keV}$ is still debated. Paizis et al. (2006a) proposed for the first time a qualitative unified physical scenario to explain the spectral evolution of NS LMXBs, including the peculiar transient hard tail. As a following step, in the quest to study in a quantitative way the evolution of the parameters describing the innermost physical conditions of NS LMXBs, a new Comptonization model was developed (compTB ${ }^{5}$, Farinelli et al., 2008), and successfully applied to a sample of bright NS LMXBs studied with INTEGRAL (Mainardi et al., 2010).

\subsection{Rapid $(\sim 100 s)$ variability}

In the archive, $100 \mathrm{~s}$ bin light-curves in the $17-30 \mathrm{keV}$ band are available for all the sources detected in the IMA part ( $2 \mathrm{ks}$ pointing basis) as well for sources of interest. Among others, we have extracted a wholearchive light-curve for the high mass X-ray binary $4 \mathrm{U} 1700-377$, for a total of $18 \mathrm{Ms}$, sampled on a $100 \mathrm{~s}$ basis. Two different time zooms of the obtained lightcurve ( 2 months, 0.8 days) are shown in Fig. 5. This
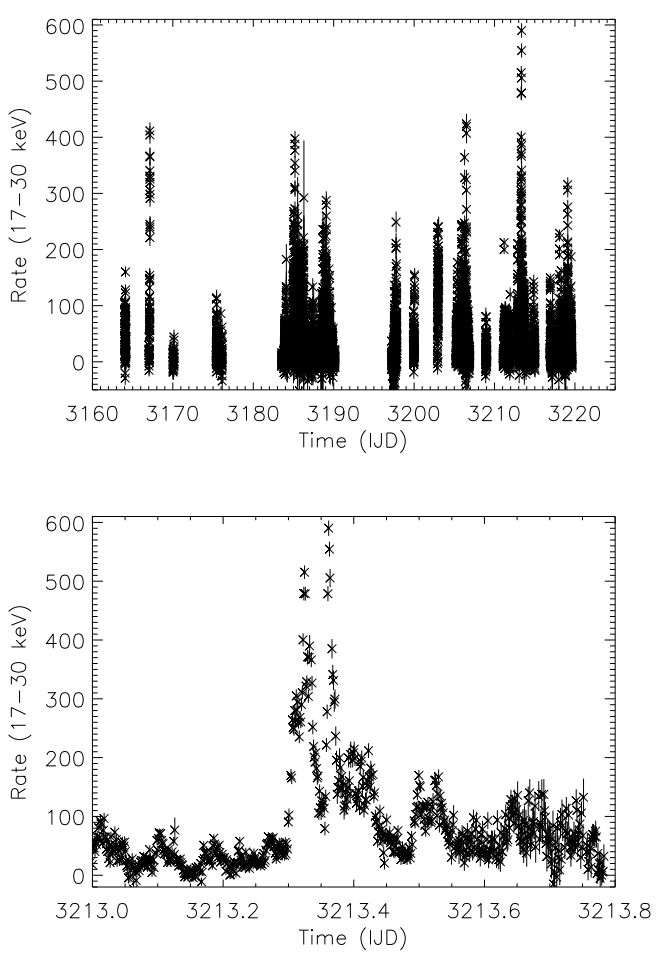

Figure 5: 17-30 keV, $100 \mathrm{~s}$ bin light-curve obtained for 4U 1700-377. Upper panel: 2 month zoom. Lower panel: 0.8 day zoom. 1 Crab corresponds to a rate of about 173 counts/s in the same band.

\footnotetext{
${ }^{5}$ http://heasarc.gsfc.nasa.gov/docs/xanadu/xspec/models/comptb.html
} 
Where do you want to zoom?

Enter R.A. [degrees]? 254.77

Enter DEC [degrees]? -15.2630

Enter size [degrees]? 0.1

IDL>

Triangles: sources with detsig(17-30keV) $>7$ Squares: sources with detsig(30-50keV) $>7$ Crosses: sources with detsig(17-50keV) $>7$ Circles : sources with detsig $(50-100 \mathrm{keV})>7$

Select the energy band $<1 / 2 / 3 / 4>$ :

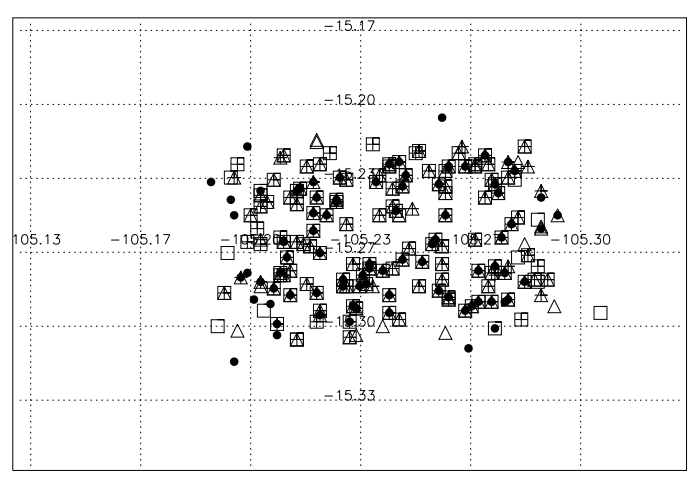

Figure 6: Output of readnew: on-screen text (upper panel) and visualization of multiple detections of the recently discovered transient MAXI J1659-152 (lower panel). See Section 3.4.

complete light-curve is the starting point for a detailed study of the fast variability over 8.5 years of INTEGRAL data in this peculiar source (Sidoli \& Paizis, in preparation).

\subsection{New Sources}

As discussed in Section 2.2, we have developed an interactive tool that reads the NEW source ASCII files created by ima_pick, grouping them by coordinates (readnew).

Fig. 6, lower panel, shows multiple detections (from different bands and pointings) of a new source, the recently discovered transient MAXI J1659-152 (a Black Hole candidate, Negoro et al., 2010, 2012), not yet included in the INTEGRAL General Reference Catalogue, hence labeled as new, NEW_1, by the software. Fig. 6, upper panel (text), shows some stages of the interactive process, with the description of the different symbols and region of the sky selected. A zoomed-in IBIS/ISGRI image of one of the multiple pointings of Fig. 6 can be seen in Fig. 7 where MAXI J1659-152 is labelled as NEW_1 by the software. The well known NS LMXB Sco $\mathrm{X}-1, \sim 9^{\circ}$ apart, is also visible and appears much softer than MAXI J1659-152: indeed, while in the softer band Sco $\mathrm{X}-1$ is the brightest source of the field (left panel, 17-30 keV), in the harder band Sco X1 is not even detected (detection significance about 2), while MAXI J1659-152 is still clearly there (detection significance about $10,50-100 \mathrm{keV}$, right panel).

We are able to trace back in the INTEGRAL/IBIS archive sources that were discovered recently (i.e. later than the creation of the INTEGRAL General Reference Catalogue used, 2010 April 8th ${ }^{6}$ ), as well as to discover previously unnoticed NEW INTEGRAL sources, such as IGR J15283-4443, discovered by our systematic analysis of all INTEGRAL/IBIS public data (Paizis et al., 2006b) and later on confirmed by $S$ wift follow-up observations (Rodriguez et al., 2010).

\section{Build your own GOLIA}

We report the main characteristics of GOLIA (disk space, costs, time employed, etc.), in order to give a quantitative idea of the effort involved in such a work. The whole analysis has been performed on a single server HP ML330G6, openSUSE 11.4, two processors Xenon Quad-Core E5506, 2.13 GHz and RAM 8GB (June 2011 cost $\sim 2300$ Euro). A total of 12 external disks of $1 \mathrm{~Tb}$ each have been purchased to store the data and the results (213 Euro each $\mathrm{Tb}$ ). Currently a total of 83733 pointings have been analyzed, i.e. starting revolution 0026 (December 2002) to 1079 (August 2011), for a total of 8.5 years of data. Disk space is needed for the raw data while the results themselves occupy a smaller fraction $(\sim 1.3 \mathrm{~Tb}$ for 8.5 years of results versus about $4.8 \mathrm{~Tb}$ of 8.5 years of data - excluding SPI raw data).

The integrated IBIS/ISGRI analysis time of the 8.5 years of data has been about 8 months with one server (roughly half of which for the IMA part and half for the LCR one). More servers (that can be used in parallel with GRID, the analysis script modular structure allows it) will result in a much lower processing time. The addition of further data products (more energy or time bins, spectral extraction or inclusion of other instruments) will of course increase the CPU time as well as disk space needed.

We have decided to begin extracting the results for IBIS/ISGRI because we believe that it is a very good starting point to investigate the less explored hard $\mathrm{X}$ ray sky (with respect to the softer JEM-X range), with

\footnotetext{
${ }^{6}$ We note that on 2012 November 15th, a new catalogue has been delivered by the ISDC, including new sources reported in the literature: http://www.isdc.unige.ch/integral/science/catalogue.
} 

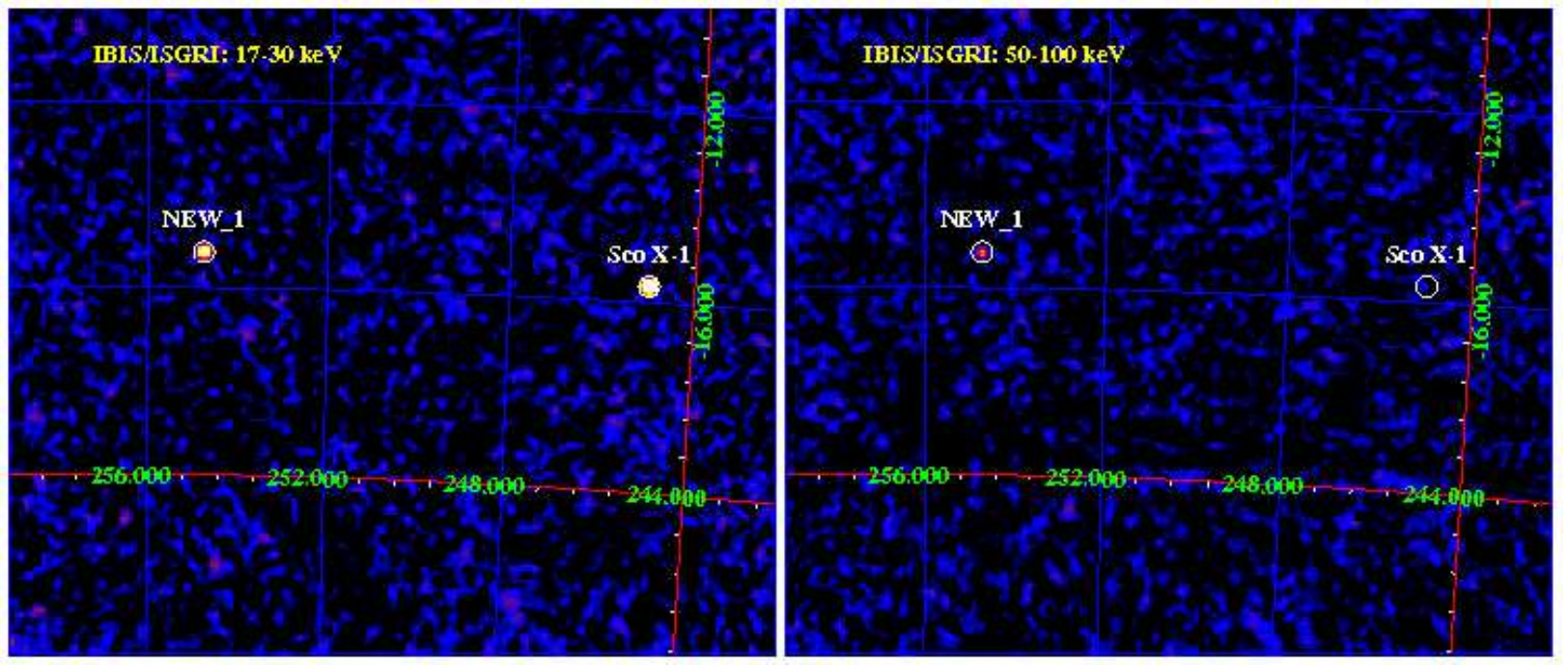

Figure 7: IBIS/ISGRI sky maps of a single pointing (ScW \#092700700010) where MAXI J1659-152 is detected by OSA and labeled as NEW (See Section 3.4).

a good (12' FWHM) angular resolution to disentangle sources in the Galactic plane and bulge (with respect to SPI, $2.5^{\circ}$ FWHM). Furthermore, we have also been triggered by our experience in the IBIS/ISGRI data analysis that allowed us to handle large datasets while choosing few energy and time bins, still capable to unveil interesting source behaviour (softening versus hardening, unexpected variabilities, transient behaviour, etc) but yet within acceptable running time.

Automatic spectral extraction, though applicable with our scripts, would have been extremely time consuming given our single-server setup. Furthermore, we decided not to perform it since the feedback with our potential users, and our previous experience on wholearchive spectra, suggested that source spectra should be re-extracted with optimized energy binning to be meaningful for a detailed analysis.

Once the setup is up and running, the maintenance of the archive is not an issue and neither is its regular update. Maps, light-curves, raw data, fits and ASCII files can all be accessed by the users, enabling a personalized usage of the archive and triggering further detailed investigations such as the ones previously described.

\subsection{Our scripts}

All the INTEGRAL public data can be freely downloaded via the ISDC dedicated web page (see footnote number 2). A tar file with the scripts we used to build GOLIA (Process and Post-process steps, i.e. the ones performed by the archive Owner, see Section 2.1 and Fig. 1) and a short description (README) can be downloaded from our GOLIA web page ${ }^{7}$. These scripts are the Shell scripts launch_IMA.sh (and its sub-routine analysis_IMA.csh), launch_LCR.sh (and its sub-routine analysis_LCR.csh) used to launch OSA looping over ScWs, and the PERL script ima_pick.pl, used to "reformat" the IMA results. Together with OSA, these are the tools we used to build GOLIA, they use freely available interpreters and need no compilation.

Once the archive is available, together with all the ASCII files created by ima_pick, browsing and visualization of the IMA results can be easily performed with any software that reads ASCII files. Mosaics of the images or collection of the 100s lightcurves can be done as described in the ISDC documentation.

\section{Conclusions}

There are several on-going international collaborations and efforts to exploit and share INTEGRAL data and results with the scientific community (to some of which we have participated as well). We recall here the Galactic bulge monitoring programme (Kuulkers et al., 2007) ${ }^{8}$, the INTEGRAL Spiral Arms collaboration ${ }^{9}$, and the INTEGRAL Galactic plane scan ${ }^{10}$, all of which deal with the analysis of IBIS/ISGRI and JEM-X data of specific sets of data (i.e. portions of the sky). Among

\footnotetext{
${ }^{7}$ http://www.iasf-milano.inaf.it/ ada/GOLIA.html

${ }^{8} \mathrm{http}: / /$ integral.esac.esa.int/BULGE/

${ }^{9}$ http://sprg.ssl.berkeley.edu/ bodaghee/isa/

${ }^{10} \mathrm{http} / / / \mathrm{gpsiasf}$. iasf-roma.inaf.it/
} 
the all sky surveys we recall the IBIS/ISGRI catalogues by Krivonos et al. (2007) ${ }^{11}$ and Bird et al. (2010). An up to date collection of all the sources discovered by $I N$ TEGRAL can be found at the INTEGRAL IGR Source page $^{12}$. Last but not least, the ISDC has developed and is maintaining an all INTEGRAL instrument archive, known as HEAVENS ${ }^{13}$ that collects light-curves, images and spectra for a given source or position in the sky, from the public data.

Notwithstanding all the above, we believe that having a complementary local archive of INTEGRAL data and products that is easy to access and to handle (be it for only one instrument) is an important way to boost scientific knowledge. It is open to suggestions and ideas of the local users in a close and frequent feedback process that maximizes the scientific outcome. Developing and maintaining a database enables complete control over what is being done and how; raw data and products are accessible at all levels (i.e. analysis steps), entire sky maps are available (not just a catalogue or a zoom on the target, but complete workable FITS files) and the results of all the detected sources can be retrieved very quickly, regardless the time interval: e.g. long term light-curves can be easily extracted also by non-INTEGRAL experts (a matter of a few seconds for the $2 \mathrm{ks}$ binning). A systematic overview of different classes of sources can be performed, stimulating new investigations and collaborations, as shown in Section 3, and enabling interesting discoveries, determination of trends, as well as providing a complementary picture to other on-going missions.

\section{Acknowledgements}

Based on observations with INTEGRAL, an ESA project with instruments and science data centre funded by ESA member states (especially the PI countries: Denmark, France, Germany, Italy, Spain, and Switzerland), Czech Republic and Poland, and with the participation of Russia and the USA. AP thanks S.E. Shaw, B.E. O'Neel and the ISDC staff since most of the knowhow needed to set-up such an archive was gained during her years at the ISDC (2000-2005). We acknowledge the Italian Space Agency financial support via contract INTEGRAL ASI-INAF I/033/10/0. We thank the anonymous Referee for his/her comments and suggestions, which improved the quality of the paper.

\footnotetext{
${ }^{11}$ http://www.mpa-garching.mpg.de/integral/survey/catalog.php

${ }^{12} \mathrm{http}: / /$ irfu.cea.fr/Sap/IGR-Sources/

${ }^{13} \mathrm{http}: / / \mathrm{www}$.isdc.unige.ch/heavens/
}

\section{References}

Bird, A.J., Bazzano, A., Bassani, L., Capitanio, F., Fiocchi, M., Hill, A.B., Malizia, A., McBride, V.A., Scaringi, S., Sguera, V., Stephen, J.B., Ubertini, P., Dean, A.J., Lebrun, F., Terrier, R., Renaud, M., Mattana, F., Götz, D., Rodriguez, J., Belanger, G., Walter, R., Winkler, C., 2010. The Fourth IBIS/ISGRI Soft Gammaray Survey Catalog. The Astrophysical Journal Supplement 186, 1-9. 0910.1704.

Courvoisier, T.J.L., Walter, R., Beckmann, V., Dean, A.J., Dubath, P., Hudec, R., Kretschmar, P., Mereghetti, S., Montmerle, T., Mowlavi, N., Paltani, S., Preite Martinez, A., Produit, N., Staubert, R., Strong, A.W., Swings, J.P., Westergaard, N.J., White, N., Winkler, C., Zdziarski, A.A., 2003. The INTEGRAL Science Data Centre (ISDC). A\&A 411, L53-L57.

Ducci, L., Sidoli, L., Mereghetti, S., Paizis, A., Romano, P., 2009. The structure of blue supergiant winds and the accretion in supergiant high-mass X-ray binaries. MNRAS 398, 2152-2165. 0906.3185.

Ducci, L., Sidoli, L., Paizis, A., 2010. INTEGRAL results on supergiant fast $\mathrm{X}$-ray transients and accretion mechanism interpretation: ionization effect and formation of transient accretion discs. MNRAS 408, 1540-1550.

Farinelli, R., Titarchuk, L., Paizis, A., Frontera, F., 2008. A New Comptonization Model for Weakly Magnetized, Accreting Neutron Stars in Low-Mass X-Ray Binaries. ApJ 680, 602-614. 0802.2639.

Krivonos, R., Revnivtsev, M., Lutovinov, A., Sazonov, S., Churazov, E., Sunyaev, R., 2007. INTEGRAL/IBIS all-sky survey in hard X-rays. A\&A 475, 775-784. arXiv: astro-ph/0701836.

Kuulkers, E., Shaw, S.E., Paizis, A., Chenevez, J., Brandt, S., Courvoisier, T.J.L., Domingo, A., Ebisawa, K., Kretschmar, P., Markwardt, C.B., Mowlavi, N., Oosterbroek, T., Orr, A., Rísquez, D., Sanchez-Fernandez, C., Wijnands, R., 2007. The INTEGRAL Galactic bulge monitoring program: the first 1.5 years. A\&A 466, 595-618. arXiv: astro-ph/0701244.

Labanti, C., Di Cocco, G., Ferro, G., Gianotti, F., Mauri, A., Rossi, E., Stephen, J.B., Traci, A., Trifoglio, M., 2003. The Ibis-Picsit detector onboard Integral. A\&A 411, L149-L152.

Lebrun, F., Leray, J.P., Lavocat, P., Crétolle, J., Arquès, M., Blondel, C., Bonnin, C., Bouère, A., Cara, C., Chaleil, T., Daly, F., Desages, F., Dzitko, H., Horeau, B., Laurent, P., Limousin, O., Mathy, F., Mauguen, V., Meignier, F., Molinié, F., Poindron, E., Rouger, M., Sauvageon, A., Tourrette, T., 2003. ISGRI: The INTEGRAL Soft Gamma-Ray Imager. A\&A 411, L141-L148.

Lubiński, P., Cadolle Bel, M., von Kienlin, A., Budtz-Jorgensen, C., McBreen, B., Kretschmar, P., Hermsen, W., Shtykovsky, P., 2005. IGR J11215-5952 discovered in INTEGRAL Galactic Plane Scans. The Astronomer's Telegram 469.

Lund, N., Budtz-Jørgensen, C., Westergaard, N.J., Brandt, S., Rasmussen, I.L., Hornstrup, A., Oxborrow, C.A., Chenevez, J., Jensen, P.A., Laursen, S., Andersen, K.H., Mogensen, P.B., Rasmussen, I., Omø, K., Pedersen, S.M., Polny, J., Andersson, H., Andersson, T., Kämäräinen, V., Vilhu, O., Huovelin, J., Maisala, S., Morawski, M., Juchnikowski, G., Costa, E., Feroci, M., Rubini, A., Rapisarda, M., Morelli, E., Carassiti, V., Frontera, F., Pelliciari, C., Loffredo, G., Martínez Núñez, S., Reglero, V., Velasco, T., Larsson, S., Svensson, R., Zdziarski, A.A., Castro-Tirado, A., Attina, P., Goria, M., Giulianelli, G., Cordero, F., Rezazad, M., Schmidt, M., Carli, R., Gomez, C., Jensen, P.L., Sarri, G., Tiemon, A., Orr, A., Much, R., Kretschmar, P., Schnopper, H.W., 2003. JEM-X: The X-ray monitor aboard INTEGRAL. A\&A 411, L231-L238.

Mainardi, L.I., Paizis, A., Farinelli, R., Kuulkers, E., Rodriguez, J., Hannikainen, D., Savolainen, P., Piraino, S., Bazzano, A., Santangelo, A., 2010. Spectral evolution of bright NS LMXBs with IN- 
TEGRAL: an application of the thermal plus bulk Comptonization model. A\&A 512, A57. 0912.2707.

Mas-Hesse, J.M., Giménez, A., Culhane, J.L., Jamar, C., McBreen, B., Torra, J., Hudec, R., Fabregat, J., Meurs, E., Swings, J.P., Alcacera, M.A., Balado, A., Beiztegui, R., Belenguer, T., Bradley, L., Caballero, M.D., Cabo, P., Defise, J.M., Díaz, E., Domingo, A., Figueras, F., Figueroa, I., Hanlon, L., Hroch, F., Hudcova, V., García, T., Jordan, B., Jordi, C., Kretschmar, P., Laviada, C., March, M., Martín, E., Mazy, E., Menéndez, M., Mi, J.M., de Miguel, E., Muñoz, T., Nolan, K., Olmedo, R., Plesseria, J.Y., Polcar, J., Reina, M., Renotte, E., Rochus, P., Sánchez, A., San Martín, J.C., Smith, A., Soldan, J., Thomas, P., Timón, V., Walton, D., 2003. OMC: An Optical Monitoring Camera for INTEGRAL. Instrument description and performance. A\&A 411, L261-L268.

Negoro, H., Kawai, N., Yamaoka, K., MAXI Team, 2012. Discoveries of new black hole transients MAXI J1659-152 and MAXI J1543-564, in: Petre, R., Mitsuda, K., Angelini, L. (Eds.), American Institute of Physics Conference Series, pp. 314-315.

Negoro, H., Yamaoka, K., Nakahira, S., Kawasaki, K., Ueno, S., Tomida, H., Kohama, M., Ishikawa, M., Mihara, T., Nakagawa, Y.E., Sugizaki, M., Serino, M., Yamamoto, T., Sootome, T., Matsuoka, M., Kawai, N., Morii, M., Sugimori, K., Usui, R., Yoshida, A., Tsunemi, H., Kimura, M., Nakajima, M., Ozawa, H., Suwa, F., Ueda, Y., Isobe, N., Eguchi, S., Hiroi, K., Daikyuji, A., Uzawa, Yamazaki, K., Matsumura, K., 2010. MAXI/GSC detection of a new hard X-ray transient source MAXI J1659-152. The Astronomer's Telegram 2873.

Paizis, A., Farinelli, R., Titarchuk, L., Courvoisier, T.J.L., Bazzano, A., Beckmann, V., Frontera, F., Goldoni, P., Kuulkers, E., Mereghetti, S., Rodriguez, J., Vilhu, O., 2006a. Average hard X-ray emission from NS LMXBs: observational evidence of different spectral states in NS LMXBs. A\&A 459, 187. arXiv: astro-ph/0607592.

Paizis, A., Gotz, D., Sidoli, L., Vercellone, S., Mereghetti, S., 2006 b. Three new INTEGRAL sources: IGR J10043-8702, IGR J105006410 and IGR J15283-4443. The Astronomer's Telegram .

Rodriguez, J., Tomsick, J.A., Bodaghee, A., 2010. Swift followup observations of 13 INTEGRAL sources. A\&A 517, A14. 1003.3741.

Sidoli, L., Paizis, A., Mereghetti, S., 2006. IGR J11215-5952: a hard X-ray transient displaying recurrent outbursts. A\&A 450, L9-L12. arXiv: astro-ph/0603081.

Sidoli, L., Romano, P., Mereghetti, S., Paizis, A., Vercellone, S., Mangano, V., Götz, D., 2007. An alternative hypothesis for the outburst mechanism in supergiant fast X-ray transients: the case of IGR J11215-5952. A\&A 476, 1307-1315. 0710.1175.

Ubertini, P., Lebrun, F., Di Cocco, G., Bazzano, A., Bird, A.J., Broenstad, K., Goldwurm, A., La Rosa, G., Labanti, C., Laurent, P., Mirabel, I.F., Quadrini, E.M., Ramsey, B., Reglero, V., Sabau, L., Sacco, B., Staubert, R., Vigroux, L., Weisskopf, M.C., Zdziarski, A.A., 2003. IBIS: The Imager on-board INTEGRAL. A\&A 411, L131-L139.

Vedrenne, G., Roques, J.P., Schönfelder, V., Mandrou, P., Lichti, G.G., von Kienlin, A., Cordier, B., Schanne, S., Knödlseder, J., Skinner, G., Jean, P., Sanchez, F., Caraveo, P., Teegarden, B., von Ballmoos, P., Bouchet, L., Paul, P., Matteson, J., Boggs, S., Wunderer, C., Leleux, P., Weidenspointner, G., Durouchoux, P., Diehl, R., Strong, A., Cassé, M., Clair, M.A., André, Y., 2003. SPI: The spectrometer aboard INTEGRAL. A\&A 411, L63-L70.

Winkler, C., Courvoisier, T.J.L., Di Cocco, G., Gehrels, N., Giménez, A., Grebenev, S., Hermsen, W., Mas-Hesse, J.M., Lebrun, F., Lund, N., Palumbo, G.G.C., Paul, J., Roques, J.P., Schnopper, H., Schönfelder, V., Sunyaev, R., Teegarden, B., Ubertini, P., Vedrenne, G., Dean, A.J., 2003. The INTEGRAL mission. A\&A 411, L1-L6.
Winkler, C., Diehl, R., Ubertini, P., Wilms, J., 2011. INTEGRAL: Science Highlights and Future Prospects. Space Science Reviews 161, 149-177. 1110.6279. 